\title{
The Quest for Broadband Seismic Data on Land
}

\author{
C. J Criss - INOVA
}

\begin{abstract}
Copyright 2019, SBGf - Sociedade Brasileira de Geofísica
This paper was prepared for presentation during the $16^{\text {th }}$ International Congress of the Brazilian Geophysical Society held in Rio de Janeiro, Brazil, 19-22 August 2019.

Contents of this paper were reviewed by the Technical Committee of the $16^{\text {th }}$ International Congress of the Brazilian Geophysical Society and do not necessarily represent any position of the SBGf, its officers or members. Electronic reproduction or storage of any part of this paper for commercial purposes without the written consent of the Brazilian Geophysical Society is prohibited.
\end{abstract}

\section{Introduction}

Broadband seismic data on land has long been the goal of geoscientists. Defined as seismic data that spans at least 6 octaves, it remains an elusive challenge in most regions of the world. In recent years the industry has successfully pushed the low frequency portion of the equation realistically to around $2-3 \mathrm{~Hz}$ but consistent generation and recording of lower frequencies continues to be a challenge. If we are to reach the final goal of 6 octaves, improvements must also be made on the high frequency portion of the spectrum. High density, single point recording is showing great promise in this area. Lighter, less expensive seismic recording equipment and improved field techniques are now enabling project designs which were previously not considered commercially viable. Projects are no longer limited by the recording systems in terms of channel count or traditional approaches to geometry design. This paper will explore the current status of source technology and recording systems from the perspective of broadband survey design and implementation, and show how modern acquisition methods can yield broader bandwidth results economically.

\section{Modern Look at Survey Design}

The traditional approach to survey design involves several steps, usually calculating required sampling intervals, migration apertures, offsets and the needed fold. The obvious trend observed in more current projects is that sampling density has increased beyond what might be required to achieve calculated frequency and dip antialiasing requirements. Boosting the trace density of projects has historically always paid dividends when it comes to the geophysical usefulness of the resultant seismic data. It is a common misconception that high fold is directly related to high sampling density; projects can achieve one goal without necessarily achieving the other. Fold becomes a relatively unimportant metric since fold is going to be high on any high density designs. What enhanced methods are demonstrating with a growing volume of evidence is that more unique source and receiver pairs in a project yield better data than multiple shots at a source point or the collection of multiple receivers in an array at a receiver point. Arrays can increase apparent $\mathrm{S} / \mathrm{N}$ but attenuate high frequencies and while multiple sweeps at a single point increase source effort they also generate redundant sampling of both signal and noise at the same proportional rate. Dense point-source and point-receiver geometries appear to be the way forward to better data. The constant advancement is recording equipment design and the resultant changes in field methods have enabled enhanced results in nearly every region of the globe. The seismic industry has realized a dramatic evolution of efficiency. In the early 1990's a project in the Middle East might be expected to gather 40 fold data into 25 meter bins while in 2017 a project in the same region would typically achieve 2,000 fold into 12.5 meter bins. And throughout this process of improved technology, operations and efficiency, we have seen dramatic reductions in cost per trace, an often overlooked metric in our industry.

\section{High Fold vs. High Density}

The combined parameters of station density, source

$$
\begin{aligned}
& \text { Bin Size Equation } \\
& \text { Bin Size }=\frac{\text { Interval Velocity }}{4 * \text { frequency } * \sin (\text { dip })} \\
& \text { frequency= unaliased maximum frequency } \\
& \text { dip }=\text { maximum expected dip }
\end{aligned}
$$

density and offset are needed to calculate the fold of a survey. Calculating density is only a function of station density and source density. A typical approach to survey design generally required the geophysicist to start with the required sub-surface sampling interval needed to image the prospect of interest. This involved calculating a bin size with the standard equation which includes parameters of velocity, signal frequency and geologic dip. This equation commonly yields results in the 25-50 meter range in most regions. Only when higher frequencies (greater than $100 \mathrm{~Hz}$ ) are required or the geologic dip is extreme (greater than 30 degrees) will this equation result in bin size requirements of 10-15 meters or less, yet it is not uncommon to find project parameters with bin sizes of 15 meters where the geologic dip is less than 30 degrees and the vibroseis sweep is limited to $60-80 \mathrm{~Hz}$. Clearly other factors weigh on the decision to sample with high density parameters. Noise estimation and noise reduction are additional key motivations for greater sampling. Many noise reduction methods directed at ground-roll and other source generated noise benefit greatly from proper sampling of the noise. Other noises, like scattering from 
the near surface are not solved by sweeping multiple times at a single point or by shooting with large arrays. Solving these issues has directed us towards single source and point receiver acquisition strategies and will continue to motivate us on future projects to design geometries that generate more unique source and receiver trace pairs rather than great quantities of data which is summed prior to processing.

\section{Enabling Single Point Recording}

Most practitioners in the industry are aware of the fact that cable-based systems have grown lighter, less expensive and less power hungry over the previous decade. This in turn has led to systems which are capable of managing live channel counts greater than 100,000 channels. A great deal of the channel count increase has been driven by the high productivity shooting methods employed in open terrain regions like the ones in the Middle East and North Africa. If you consider the limitations of cable based recording systems our industry may now be close to the last generation of cable-based technology. Cable-based systems require power transmission and data transmission through the ground network. The need for distributed power constrains today's cable-based designs to copper cables. These cables will define the limit to how low the price per channel can get and ultimately the number of channels which can be practically implemented. On a large project with 50,000 channels the weight of the cable easily exceeds $100,000 \mathrm{~kg}$ and around $70 \%$ of the total system weight. The benefits to this method are still important, real time data quality control and management are the dividends of the cable-based approach and why it remains the most popular method of acquisition.

Cable-less technology is now in its third generation. The smart-geophone concept that manages its own absolute timing and recording of data is a reality which will have a profound impact on future survey designs. Units that weigh around $0.5 \mathrm{~kg}$ that can record continuous seismic data for 50 days or more with greater fidelity than current cable systems are now commercial. The potential benefits are many. Project geometries are not going to be limited by channel count, station densities and any station spacing is possible including variable density designs. Since third generation nodes are totally autonomous, they can be used to augment any

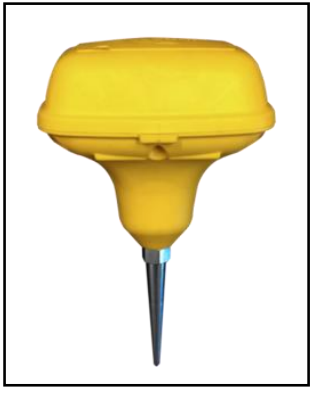

Figure 1 smart geophone technology represents the third generation cable-less system. project in a hybrid approach. It should become common for any seismic crew to have cable-less nodes to add station density when required or to deploy in difficult situations. Nodes have demonstrated yield rates exceeding $99 \%$ making them more effective at trace yields than modern cable-based systems. By virtue of the cable-less architecture, high density single point acquisition is more cost effective than shooting with cables simply because less equipment needs to be handled in the field. Cable-less technology acceptance has grown constantly since the early 2000's and in some regions of the world like North America acceptance is virtually $100 \%$.

\section{Conclusions}

The goals for seismic projects have evolved dramatically with the increased capability of seismic recording equipment. Over sampling with dense geometries that were considered impossible just 10 years ago has become normal in some areas. Cable-based architectures have responded to demands but may be approaching the limit on what can be achieved with current technologies. The cost of the cables themselves will prevent the cost per channel of these types of systems from going lower no matter how many channels are deployed. Cable-less technology is uniquely positioned to move seismic acquisition projects to the next level of fidelity and high density sampling. Unrestrained by the cables, virtually any seismic geometry strategy is possible and cable-less systems will play a major role in gaining the next step change in the affordability of ultra-high density seismic projects.

\section{References}

Berni, A. J,. and W. L. Roever, 1989, Field array performance: Theoretical study of spatially correlated variations in amplitude coupling and static shift and case study: Geophysics, 54, 451-459.

Levin, F. K., 1989, The effect of geophone arrays on random noise: Geophysics, 54, 1466-1473. 